\title{
Conocimientos, actitudes y conductas sobre sexualidad en estudiantes de educación superior del estado de Aguascalientes
}

\author{
Arias-Ulloa $R^{*}$, Valencia-García $V^{* *}$, Prado-Aguilar $C A^{* * *}$, Jiménez-Cetina SE***
}

\section{Resumen}

- Objetivo. Determinar los conocimientos, actitudes y - conductas sobre sexualidad en estudiantes de edu- cación superior del estado de Aguascalientes. Meto- dología. Estudio observacional descriptivo, tipo encues-

- ta transversal, mediante muestreo probabilístico por

- conglomerados monoetápico. Se analizaron 1,134

- encuestas. Resultados: El $41.5 \%$ de las mujeres y el

- $58.5 \%$ de los hombres tienen vida sexual activa, co-

- menzando las primeras a los 18 años en promedio y

- los segundos a los 17. Los varones han tenido el doble

- de parejas sexuales que las mujeres (4.44/2.12). Se

- detectó que sólo el $70.1 \%$ de los estudiantes utilizó

- el condón en su primera relación sexual, el resto de

- los jóvenes pueden tener riesgos reproductivo como

- embarazos no deseados, abortos clandestinos e infec-

- ciones de transmisión sexual; además, se encontraron

- riesgos en el uso de drogas durante el acto sexual,

- fuentes poco confiables de obtención de información

- sobre sexualidad, así como en los deficientes conoci-

- mientos específicos sobre sexualidad.

- Palabras clave: Conocimientos sobre sexualidad, actitu- des sobre sexualidad, conductas sobre sexualidad, relaciones sexuales, parejas sexuales, estudiantes universitarios.

\section{Abstract}

Objective. To determine the knowledge, attitudes and conducts in university students from the state of Aguascalientes. Methodology. Descriptive crosssectional observational survey, by means of monoetapic probabilistic sampling by conglomerates. I, I 34 surveys were analyzed. Results: The 41.5\% of the women and $58.5 \%$ of the men already have active sexual life, beginning the first the 18 years in average and the seconds to the 17. The men have had the double of sexual pairs that the women (4.44/2.12). It was detected that only the $70.1 \%$ of the students in the first sexual intercourse used condom, what means specific risks for diseases of sexual transmission and abortion; in addition, risks in the drug use were during sexual intercourse and sources of sex-related information, as well as in the specific sexual knowledge.

Key words: Sexual knowledge, sexual attitudes, sexual conducts, sexual intercourse, sexual pairs, university students.

* Investigación financiada por el Instituto Nacional de Desarrollo Social de la Secretaría de Desarrollo Social del Gobierno Federal. México.

(Profesor - Investigador. Departamento de Salud Pública. Centro de Ciencias Biomédicas. Universidad Autónoma de Aguascalientes. Tel. y Fax. 9107400 Ext. 405. E-mail: rarias@correo.uaa.mx

** Centro de Capacitación para el Desarrollo Comunitario A.C. Tel. y Fax. 9932984 E mail: cecadec@prodigy.netmx

*** Profesor - Investigador. Departamento de Salud Pública. Centro de Ciencias Biomédicas. Universidad Autónoma de Aguascalientes. Tel. y Fax. 91084 44. E mail: carlospa@uiessags.com

**** Auditor Médico. Subdirección de Auditoría Médica. Instituto de Salud del Estado de Aguascalientes. Tel. 9107900 ext. 7270. E mail:dra_elijimenezc@yahoo.com.mx 


\section{Introducción}

El ejercicio de la sexualidad al final de la adolescencia y principios de la edad adulta se ha convertido en un problema preocupante para las autoridades de salud pública. Es en esta etapa de la vida donde se inician las relaciones sexuales, con los consiguientes riesgos que ello implica: transmisión de infecciones por vía sexual, embarazos no planeados, abortos clandestinos, entre otros. ${ }^{1}$

La población de estudiantes de educación superior comparte estos mismos riesgos al encontrarse en esos grupos de edad, con características muy peculiares, pues son influidos por su contexto social, los grupos donde se desenvuelven son diversos, así como sus circunstancias económicas y culturales. ${ }^{2,3}$

Diversos estudios $4,5,6,7,8$ han mostrado la vulnerabilidad de los jóvenes estudiantes en el ejercicio de su sexualidad, pues una buena proporción no utiliza métodos anticonceptivos en sus relaciones sexuales, quedando en riesgo de adquirir alguna enfermedad de transmisión sexual, embarazo no deseado, e incluso llegando a abortar el producto. Asimismo, las fuentes de obtención de información sobre este tema no son las adecuadas.

En nuestro país, los jóvenes no escapan a esta realidad, según la Encuesta Nacional de la Juventud $2005^{9}$, poco más del $48 \%$ afirmó tener relaciones sexuales, $52 \%$ de ellos iniciaron su vida sexual activa con su novio(a), pero un $2.5 \%$ refirió haberlo hecho con un sexo servidor(a). El 92\% de los jóvenes mencionó conocer los métodos anticonceptivos, pero aproximadamente $36 \%$ no los utiliza. La Encuesta de Salud Sexual y Reproductiva ${ }^{10}$ aplicada en el año 2007 en el Distrito Federal señala que el 43.8\% de los jóvenes tuvieron su primera relación sexual entre los 13 y 15 años. El 63\% de ellos dijo que inició su vida sexual activa con su novio(a), mientras que el $28 \%$ lo hizo con un amigo(a); casi el $6 \%$ aseveró haberlo hecho por estar bajo el influjo de bebidas alcohólicas o drogas; el $10.3 \%$ de las mujeres afirmaron haber estado embarazadas, de las cuales 14 reconocieron haber interrumpido el proceso de gestación. De igual forma, este estudio reveló que el $94 \%$ de los jóvenes conoce algún método anticonceptivo, pero sólo el $50 \%$ los utiliza, siendo el condón el más utilizado. La Encuesta Nacional de Salud $2006^{11}$ en sus resultados para el estado de Aguascalientes establece que el 39\% de los adultos entre 20 y 29 años de edad utilizan algún método de planificación familiar, siendo las mujeres quienes mayormente lo hacen.

Finalmente, otro estudio realizado por la Universidad Autónoma del Estado de México ${ }^{12}$ sobre la salud sexual y reproductiva de los adolescentes en México presenta resultados similares a los anteriores, pero enfatiza que los resultados muestran claramente el hueco entre la política y la práctica, lo que contribuye a impedir el uso del condón entre la juventud mexicana.

Todos los estudios referidos anteriormente muestran resultados para la población de adolescentes en general, sin especificar cómo 
se comporta el fenómeno entre población estudiantil de nivel superior, en donde se esperaría que por el nivel de estudios tuviera un comportamiento favorable en conocimientos, actitudes y conductas sobre el ejercicio de la sexualidad.

El objetivo de este estudio fue determinar los conocimientos, actitudes y conductas sobre sexualidad en estudiantes de educación superior del estado de Aguascalientes, haciendo un análisis de los resultados por sexo.

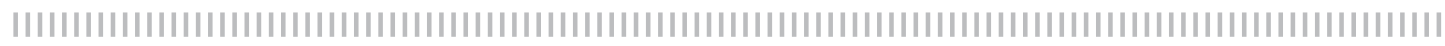

\section{Material y métodos}

Se realizó un estudio observacional descriptivo de tipo encuesta transversal, mediante muestreo probabilístico por conglomerados monoetápico.

El universo estuvo constituido por 19,902 estudiantes de nueve instituciones de educación superior agrupadas en el seno del Consejo Interuniversitario contra las Adicciones del estado de Aguascalientes que dieron su anuencia para participar en el estudio.

Se validaron y analizaron 1,134 encuestas dando una confiabilidad del $95 \%$ y una precisión del $2.8 \%$. El error de muestreo fue de $0.01 \%$, con un intervalo de confianza para el $95 \%$ de los datos de la variable "tener relaciones sexuales" entre 47.04\% y $50.96 \%$. La determinación del tamaño de muestra y la selección muestral se realizó mediante el paquete estadístico EpiDat ${ }^{r}$ desarrollado por la Organización Panamericana de la Salud y la Xunta de Andalucía, España, considerando como unidad mues- tral al grupo y como unidad de análisis el individuo.

Se diseñó un instrumento para recolección de datos tipo cuestionario autoadministrado que constó de 40 ítems, abordando aspectos generales del individuo, relaciones sexuales y su contexto, uso de métodos anticonceptivos y conocimientos específicos sobre el ejercicio de su sexualidad; el instrumento fue validado por seis expertos siguiendo la metodología de la Corporación RAND (Research and Development). Por ser un estudio donde no se pone en riesgo la integridad ni la salud del sujeto de estudio, no se solicitó el consentimiento informado por escrito, pero si se garantizó el anonimato y la confidencialidad de las respuestas. La aplicación de la encuesta se realizó en grupo para incrementar la confiabilidad de las respuestas. Se generó una base de datos utilizando el paquete estadístico SPSS(r) versión 17 en español, con el que se obtuvieron estadísticas descriptivas e inferenciales.

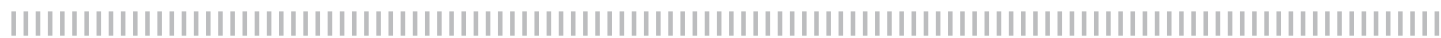

\section{Resultados}

El análisis de los datos arrojó que el $53 \%$ de los encuestados pertenece al género masculino y el $47 \%$ al femenino. El promedio de edad en ambos grupos fue de 21 años con una desviación estándar de 2.793, con una moda de 18 años para los varones y 20 para las mujeres.

El 5.6\% manifestó tener hijos; de éstos, el 61.9\% afirmó que en promedio 1.05 de sus hijos no estuvo planeado.

Una variable de sumo interés es el status laboral de los estudiantes de educación superior, pues ello refleja el esfuerzo adicional que deben realizar para continuar con sus estudios. A este respecto el $40.7 \%$ tiene un trabajo remunerado, el resto se dedica exclusivamente al estudio. 
Al contrastar las variables tienes hijos y status laboral, se encontró una diferencia estadísticamente significativa con una po- sibilidad 3.19 veces mayor de que quienes tienen hijos y estudian, deben trabajar. La tabla 1 muestra estos resultados.

\section{Tabla I}

\section{Status laboral y tenencia de hijos en estudiantes de educación superior del estado de Aguascalientes}

\begin{tabular}{|c|c|c|c|c|}
\hline \multirow[b]{2}{*}{$\begin{array}{l}\text { Además de estudiar } \\
\text { ¿también trabajas? }\end{array}$} & \multicolumn{4}{|c|}{ ¿Tienes hijos? } \\
\hline & $\mathrm{Si}$ & $\mathbf{S i}$ & $\begin{array}{l}\text { No } \\
418 \\
\end{array}$ & $\begin{array}{c}\text { Total } \\
461 \\
\end{array}$ \\
\hline & No & 21 & 652 & 673 \\
\hline & Total & 64 & 1070 & 1134 \\
\hline $2=19.795(p<0.05)$ & \multicolumn{4}{|c|}{$\mathrm{OR}=3.194(\mathrm{IC95} \% 1.89-5.459)$} \\
\hline
\end{tabular}

Un dato que llama la atención es que sólo el $17 \%$ aseguró que en su institución educativa se dan clases o se brinda consejería sobre sexualidad; el $35.4 \%$ dijo que no y el $47.5 \%$ manifestó no saber si en su escuela se brindaba este tipo de asesoría.

Cuando se indagó cuál era la fuente principal de información por la que adquieren conocimientos sobre sexualidad, se observó que existe una relación inversa entre las fuentes de donde recibieron la información y de las que les hubiera gustado recibirla. La gráfica 1 muestra los porcentajes para las dos preguntas.
La pregunta central de la investigación fue si los jóvenes estudiantes han tenido relaciones sexuales por lo menos alguna vez en la vida. El 50.8\% respondió que nunca; el $7.1 \%$ refirió haberlas tenido una vez y el $42.1 \%$ aseguró haber tenido relaciones sexuales más de una vez.

Al hacer el análisis por sexo, encontramos diferencias estadísticamente significativas; el $58.5 \%$ de los hombres han tenido relaciones por lo menos una vez, en contraste con $41.5 \%$ de las mujeres. La tabla 2 muestra los resultados.

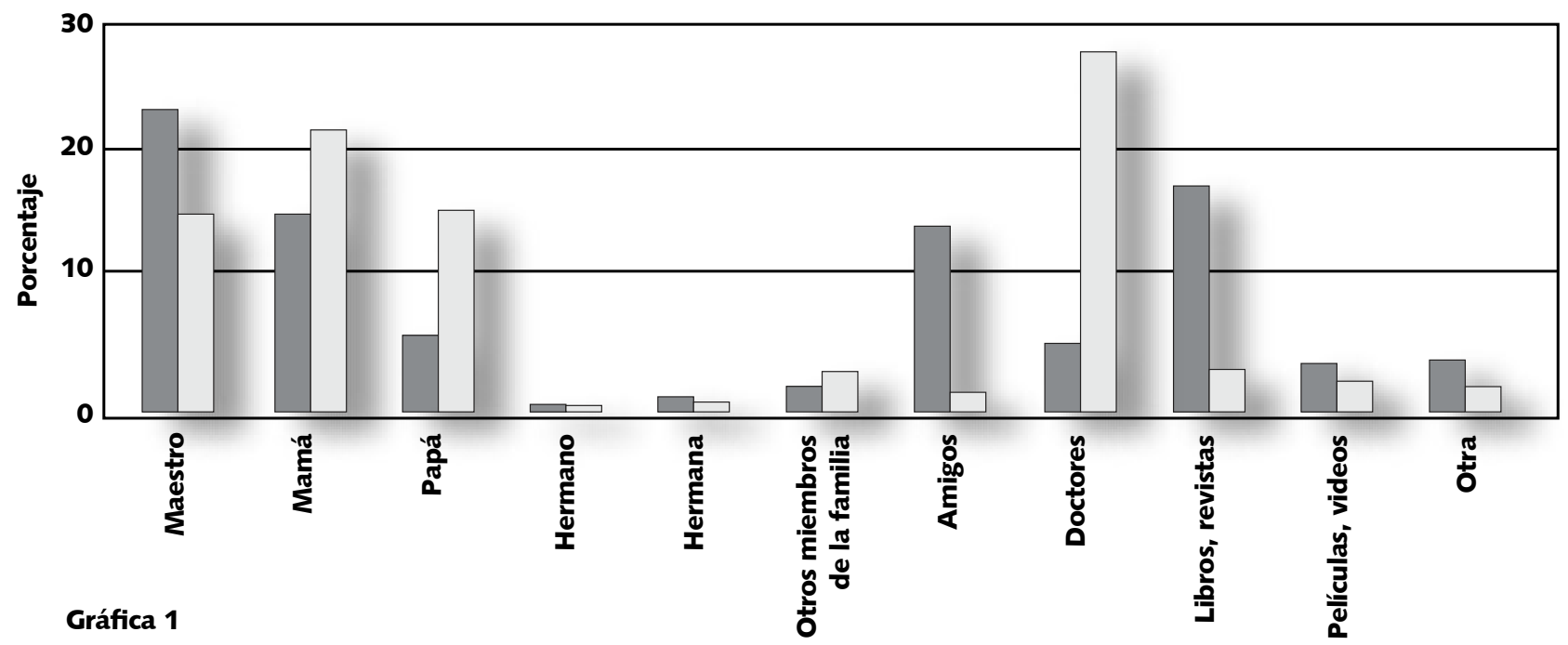

¿Cuál ha sido la fuente más importante por la que has adquirido conocimientos acerca de la sexualidad? ¿De quién o de dónde te hubiera gustado recibir más información sobre sexualidad? 


\section{Tabla 2}

Vida sexual activa según sexo en estudiantes de educación superior del estado de Aguascalientes

\begin{tabular}{|c|c|c|c|c|c|}
\hline \multirow[b]{2}{*}{ ¿Cuál es tu sexo? } & \multicolumn{5}{|c|}{$\begin{array}{l}\text { ¿Has tenido relaciones sexuales } \\
\text { (te has acostado con alguna persona) alguna vez? }\end{array}$} \\
\hline & Hombre & $\begin{array}{c}\text { Nunca } \\
239 \\
41.5 \% \\
\end{array}$ & $\begin{array}{c}\text { Una vez } \\
44 \\
54.3 \% \\
\end{array}$ & $\begin{array}{c}\text { Más de una vez } \\
318 \\
66.7 \% \\
\end{array}$ & $\begin{array}{c}\text { Total } \\
601 \\
53 \% \\
\end{array}$ \\
\hline & Mujer & $\begin{array}{c}337 \\
58.5 \%\end{array}$ & $\begin{array}{c}37 \\
45.7 \%\end{array}$ & $\begin{array}{c}159 \\
33.3 \%\end{array}$ & $\begin{array}{c}533 \\
47.0 \%\end{array}$ \\
\hline & Total & $\begin{array}{c}576 \\
50.8 \%\end{array}$ & $\begin{array}{c}81 \\
7.1 \% \%\end{array}$ & $\begin{array}{c}477 \\
42.1 \%\end{array}$ & $\begin{array}{r}1134 \\
100 \%\end{array}$ \\
\hline \multicolumn{6}{|c|}{$x^{2}=66.440(p<0.05)$} \\
\hline
\end{tabular}

Estos resultados nos demuestran que aproximadamente la mitad de los estudiantes ya tienen relaciones sexuales por lo que es una población en riesgo para diversos acontecimientos como embarazos no deseados, hijos no planeados, aborto clandestino, infecciones de transmisión sexual.

En general, el noviazgo fue la principal relación afectiva que los jóvenes tuvieron con su primera pareja sexual; sin embar- go, al realizar el análisis por sexo, existen diferencias estadísticamente significativas, encontrando que las mujeres preferentemente lo hacen con su novio en un $79.6 \%$, mientras que en los hombres se diversifica más, $51.7 \%$ con su novia, $39.5 \%$ con una amiga, $3.6 \%$ con una prostituta y el resto con otras personas. La tabla 3 muestra los resultados pormenorizados.

\section{Tabla 3}

Relación afectiva en la primera relación sexual según sexo en estudiantes de educación superior del estado de Aguascalientes

\begin{tabular}{|c|c|c|c|c|c|c|}
\hline \multirow[b]{3}{*}{ ¿Cuál es tu sexo? } & \multicolumn{6}{|c|}{$\begin{array}{l}\text { ¿Qué relación tenías o tienes } \\
\text { con la primera persona con } \\
\text { la que tuviste relaciones sexuales? }\end{array}$} \\
\hline & \multirow[b]{2}{*}{ Hombre } & Novio(a) & Amigo(a) & Prostituto(a) & Otra & Total \\
\hline & & $\begin{array}{c}187 \\
51.7 \% \\
\end{array}$ & $\begin{array}{c}143 \\
39.5 \% \\
\end{array}$ & $\begin{array}{r}13 \\
3.6 \\
\end{array}$ & $\begin{array}{c}19 \\
5.2 \% \\
\end{array}$ & $\begin{array}{c}362 \\
100 \% \\
\end{array}$ \\
\hline & Mujer & $\begin{array}{c}156 \\
79.6 \% \\
\end{array}$ & $\begin{array}{c}22 \\
11.2 \% \\
\end{array}$ & $\begin{array}{c}1 \\
0.5 \% \\
\end{array}$ & $\begin{array}{c}17 \\
8.7 \% \\
\end{array}$ & $\begin{array}{c}196 \\
100 \% \\
\end{array}$ \\
\hline & Total & $\begin{array}{r}343 \\
61.5 \% \\
\end{array}$ & $\begin{array}{r}165 \\
29.6 \% \\
\end{array}$ & $\begin{array}{c}14 \\
2.5 \% \\
\end{array}$ & $\begin{array}{c}36 \\
6.4 \% \\
\end{array}$ & $\begin{array}{c}558 \\
100 \% \\
\end{array}$ \\
\hline & & & & & \multicolumn{2}{|c|}{$x^{2}=57.651(p<0.05$} \\
\hline
\end{tabular}

El promedio de edad en la cual los estudiantes tuvieron su primera relación sexual fue de 17.57 años con una desviación estándar de 2.317 años. Al desglosar por sexo este resultado, los hombres la tuvieron a los 17.19 años con una desviación estándar de 2.091 y las mujeres a los 18.28 con una desviación de 2.545 años; estas cifras resultaron estadísticamente significativas al comparar los promedios por sexo $(F=29.135 p<0.05)$. 
El uso del condón en la primera relación sexual muestra el grado de conocimiento y responsabilidad que los jóvenes tienen al iniciar su vida sexual. Los resultados muestran que el $70.1 \%$ de los jóvenes utilizaron este medio de protección.

Se exploró la continuidad en la vida sexual activa mediante la pregunta ¿cuántas veces has tenido relaciones sexuales en el último mes? Las respuestas mostraron que el $29.9 \%$ las ha tenido de una a dos veces; el $14.2 \%$ entre tres a cuatro veces; el $7.3 \%$ de cinco a seis veces; siete o más veces lo refirió el $9.5 \%$ y no han vuelto a tener relaciones sexuales el $39.1 \%$. No se encontraron diferencias estadísticamente significativas en la asociación por sexo.

El promedio de personas con las cuales han tenido relaciones sexuales fue de 3.62 con una desviación estándar de 5.01. Sin embargo, al hacer la discriminación por sexo, se observa que los hombres han tenido el doble de personas con las que han tenido relaciones sexuales en comparación con las mujeres, en una relación de 2:1, esta relación fue estadísticamente significativa en el nivel de $p<0.05$

Al relacionar la variable anterior con la pregunta que explora la estabilidad con la pareja sexual, se encontró que casi la mitad de los estudiantes lo ha hecho con la misma pareja, el $25.2 \%$ ha cambiado alguna vez de pareja y el $27.8 \%$ ha cambiado más de una vez de pareja. El análisis por sexo demuestra que los hombres cambian con mayor frecuencia de pareja (62.2\%), lo que los sitúa en mayor riesgo en comparación con las mujeres que sólo han cambiado de pareja en el $36.2 \%\left(x^{2}=40.484\right.$ $p<0.05$ )

El uso de métodos anticonceptivos en los estudiantes que continúan con vida sexual activa mostró que el $87.6 \%$ sí los utilizan, mientras que el $12.4 \%$ no los usan regularmente. No se encontraron diferen- cias estadísticamente significativas al realizar el análisis por sexo. El método más utilizado es el condón con el $83.0 \%$; el coito interrumpido ocupó el $5.4 \%$; las pastillas anticonceptivas el $5.4 \%$; el dispositivo intrauterino el $1.1 \%$ y otro método con el $2.9 \%$.

Las razones más importantes que los jóvenes refirieron para no utilizar métodos anticonceptivos fueron: porque no me gusta $(28.1 \%)$; porque no sé cómo usarlos $(5.8 \%)$; porque es malo para la salud $(7.4 \%)$; porque no se siente lo mismo $(24.8 \%)$ y luego aparecen dispersas otras razones (33.9\%). Es de resaltar que ninguna persona consideró el no uso de métodos anticonceptivos porque va en contra de su religión.

El ejercicio irresponsable de la sexualidad puede llevar a sucesos no esperados para quien la realiza, tal es el caso de los embarazos no deseados. En este sentido se les preguntó a los estudiantes varones, si habían embarazado a alguien sin habérselo propuesto y a las mujeres si habían estado embarazadas sin proponérselo. El $7.8 \%$ de los hombres embarazó a su pareja sin habérselo propuesto, en tanto que el $15.2 \%$ de las mujeres quedó embarazada sin haberlo deseado. También se abordó el aborto como una medida para interrumpir el embarazo no deseado, el $13.6 \%$ de los varones manifestó que estaría de acuerdo en que su pareja abortara y el $6.6 \%$ de las mujeres manifestó haber abortado alguna vez en su vida.

El último aspecto que abordó la investigación fueron los conocimientos que los estudiantes tienen respecto al ejercicio de su sexualidad. Se formularon preguntas con respuestas dicotómicas de "verdadero" o "falso". Los resultados a estos ítems estratificados por sexo se muestran en la tabla 4. 


\section{Tabla 4}

Conocimientos y actitudes sobre el ejercicio de su sexualidad, según sexo, en estudiantes de educación superior del estado de Aguascalientes.

\begin{tabular}{|c|c|c|c|c|}
\hline \multirow{3}{*}{$\begin{array}{l}\text { 1. El coito interrumpido o "salirse antes" } \\
\text { es un método bastante seguro }\end{array}$} & & Verdadero & Falso & $\begin{array}{c}\text { Significación } \\
\text { estadística }\end{array}$ \\
\hline & Hombre & $\begin{array}{c}44 \\
7.3 \%\end{array}$ & $\begin{array}{c}557 \\
92.7 \%\end{array}$ & \multirow{2}{*}{$\begin{array}{l}x^{2}=1.327 \\
p>0.05\end{array}$} \\
\hline & Mujer & $\begin{array}{c}30 \\
5.6 \%\end{array}$ & $\begin{array}{c}503 \\
94.4 \% \\
\end{array}$ & \\
\hline \multirow[t]{2}{*}{$\begin{array}{l}\text { 2. Si se lava con cuidado, podemos } \\
\text { utilizar el condón varias veces }\end{array}$} & $\begin{array}{c}\text { Hombre } \\
1.8 \% \\
\end{array}$ & $\begin{array}{c}11 \\
98.2 \% \\
\end{array}$ & 590 & \multirow{2}{*}{$\begin{aligned} x^{2} & =0.950 \\
p & >0.05\end{aligned}$} \\
\hline & $\begin{array}{c}\text { Mujer } \\
1.1 \%\end{array}$ & $\begin{array}{c}6 \\
98.9 \% \\
\end{array}$ & 527 & \\
\hline $\begin{array}{l}\text { 3. Para utilizar pastillas anticonceptivas } \\
\text { es conveniente ser revisada por un médico }\end{array}$ & $\begin{array}{c}\text { Hombre } \\
\text { Mujer }\end{array}$ & $\begin{array}{c}522 \\
86.9 \% \\
487 \\
91.4 \% \\
\end{array}$ & $\begin{array}{c}79 \\
13.1 \% \\
46 \\
8.6 \% \\
\end{array}$ & $\begin{aligned} X^{2} & =5.870 \\
P & <0.05\end{aligned}$ \\
\hline $\begin{array}{l}\text { 4. Tener relaciones completas durante } \\
\text { la regla es un método seguro para evitar } \\
\text { el embarazo }\end{array}$ & $\begin{array}{l}\text { Hombre } \\
\text { Mujer }\end{array}$ & \begin{tabular}{c|}
156 \\
$26.0 \%$ \\
112 \\
$21.0 \%$ \\
\end{tabular} & \begin{tabular}{c|}
445 \\
$74.0 \%$ \\
421 \\
$79.0 \%$ \\
\end{tabular} & $\begin{aligned} x^{2} & =3.825 \\
P & =0.05\end{aligned}$ \\
\hline $\begin{array}{l}\text { 5. Las pastillas anticonceptivas son } \\
\text { eficaces si se toman antes de cada } \\
\text { relación sexual }\end{array}$ & $\begin{array}{c}\text { Hombre } \\
\text { Mujer }\end{array}$ & $\begin{array}{c}218 \\
36.3 \% \\
88 \\
16.5 \% \\
\end{array}$ & $\begin{array}{c}383 \\
63.7 \% \\
445 \\
83.5 \% \\
\end{array}$ & $\begin{aligned} & x^{2}=55.995 \\
& P<0.05\end{aligned}$ \\
\hline $\begin{array}{l}\text { 6. Tomar pastillas anticonceptivas protege } \\
\text { de adquirir enfermedades de transmisión } \\
\text { sexual (SIDA, Hepatitis B, gonorrea, etc.) }\end{array}$ & $\begin{array}{c}\text { Hombre } \\
\text { Mujer }\end{array}$ & $\begin{array}{c}23 \\
3.8 \% \\
16 \\
3.0 \% \\
\end{array}$ & $\begin{array}{c}578 \\
96.2 \% \\
517 \\
97.0 \% \\
\end{array}$ & $\begin{aligned} x^{2} & =0.579 \\
p & >0.05\end{aligned}$ \\
\hline \multirow[t]{2}{*}{$\begin{array}{l}\text { 7. El preservativo o condón protege de las } \\
\text { enfermedades de transmisión sexual }\end{array}$} & Hombre & $\begin{array}{c}538 \\
89.5 \% \\
\end{array}$ & $\begin{array}{c}63 \\
10.5 \% \\
\end{array}$ & \multirow{2}{*}{$\begin{array}{l}x^{2}=1.012 \\
p>0.05\end{array}$} \\
\hline & Mujer & $\begin{array}{c}467 \\
87.6 \%\end{array}$ & $\begin{array}{c}66 \\
12.4 \% \\
\end{array}$ & \\
\hline $\begin{array}{l}\text { 8. El SIDA no se transmite en las relaciones } \\
\text { sexuales que se limitan a la penetración bucal }\end{array}$ & Hombre & $\begin{array}{c}143 \\
23.8 \% \\
132 \\
24.8 \% \\
\end{array}$ & $\begin{array}{c}458 \\
76.2 \% \\
401 \\
75.2 \% \\
\end{array}$ & $\begin{array}{l}x^{2}=0.145 \\
p>0.05\end{array}$ \\
\hline $\begin{array}{l}\text { 9. El preservativo es seguro si se coloca } \\
\text { inmediatamente antes de "venirse" } \\
\text { o "eyacular" }\end{array}$ & $\begin{array}{l}\text { Hombre } \\
\text { Mujer }\end{array}$ & $\begin{array}{c}68 \\
11.3 \% \\
98 \\
18.4 \% \\
\end{array}$ & $\begin{array}{c}533 \\
88.7 \% \\
435 \\
81.6 \% \\
\end{array}$ & $\begin{array}{c}x^{2}=11.306 \\
P<.05\end{array}$ \\
\hline $\begin{array}{l}\text { 10. Utilizar preservativos permite tener unas } \\
\text { relaciones sexuales más seguras y placenteras }\end{array}$ & $\begin{array}{c}\text { Hombre } \\
\text { Mujer }\end{array}$ & $\begin{array}{c}460 \\
76.5 \% \\
448 \\
84.1 \% \\
\end{array}$ & $\begin{array}{c}141 \\
23.5 \% \\
85 \\
15.9 \% \\
\end{array}$ & $\begin{array}{l}X^{2}=9,993 \\
P<0.05\end{array}$ \\
\hline $\begin{array}{l}\text { 11. La píldora de emergencia es un método } \\
\text { anticonceptivo que se puede usar } \\
\text { regularmente antes de una relación sexual }\end{array}$ & $\begin{array}{c}\text { Hombre } \\
\text { Mujer }\end{array}$ & $\begin{array}{c}147 \\
24.5 \% \\
75 \\
14.1 \% \\
\end{array}$ & $\begin{array}{c}454 \\
75.5 \% \\
458 \\
85.9 \% \\
\end{array}$ & $\begin{array}{l}x^{2}=19.361 \\
P<0.05\end{array}$ \\
\hline $\begin{array}{l}\text { 12. Las mujeres tienen derecho a tener varias } \\
\text { parejas sexuales durante su vida }\end{array}$ & $\begin{array}{l}\text { Hombre } \\
\text { Mujer }\end{array}$ & $\begin{array}{c}373 \\
62.1 \% \\
360 \\
67.5 \% \\
\end{array}$ & $\begin{array}{c}228 \\
37.9 \% \\
173 \\
32.5 \% \\
\end{array}$ & $\begin{array}{l}x^{2}=3.710 \\
p>0.05\end{array}$ \\
\hline $\begin{array}{l}\text { 13. Los hombres tienen derecho a tener varias } \\
\text { parejas sexuales durante su vida }\end{array}$ & $\begin{array}{c}\text { Hombre } \\
\text { Mujer }\end{array}$ & $\begin{array}{c}434 \\
72.2 \% \\
337 \\
63.2 \%\end{array}$ & $\begin{array}{c}167 \\
27.8 \% \\
196 \\
36.8 \%\end{array}$ & $\begin{array}{l}x^{2}=10.481 \\
P<0.05\end{array}$ \\
\hline
\end{tabular}




\begin{tabular}{|c|c|c|c|c|}
\hline \multirow[t]{2}{*}{$\begin{array}{l}\text { 14. Es conveniente que las mujeres lleguen } \\
\text { vírgenes al matrimonio }\end{array}$} & Hombre & $\begin{array}{c}259 \\
43.1 \% \\
\end{array}$ & $\begin{array}{c}342 \\
56.9 \%\end{array}$ & \multirow{2}{*}{$\begin{aligned} x^{2} & =1.757 \\
p & >0.05\end{aligned}$} \\
\hline & Mujer & $\begin{array}{c}209 \\
39.2 \%\end{array}$ & $\begin{array}{c}324 \\
60.8 \%\end{array}$ & \\
\hline \multirow[t]{2}{*}{$\begin{array}{l}\text { 15. Es conveniente que los hombres lleguen } \\
\text { vírgenes al matrimonio }\end{array}$} & Hombre & $\begin{array}{c}205 \\
34.1 \% \\
\end{array}$ & $\begin{array}{c}396 \\
65.9 \% \\
\end{array}$ & \multirow{2}{*}{$\begin{aligned} x^{2} & =3.172 \\
p & >0.05\end{aligned}$} \\
\hline & Mujer & $\begin{array}{c}209 \\
39.2 \%\end{array}$ & $\begin{array}{c}324 \\
60.8 \%\end{array}$ & \\
\hline
\end{tabular}

La tabla anterior muestra que un porcentaje importante de los estudiantes tienen conocimientos y actitudes erróneos con respecto al ejercicio de su sexualidad. Destacan las proporciones de respuestas erróneas encontradas en las preguntas 4, $5,7,8,9,10,11,12,13,14$ y 15 . En las preguntas $3,5,9,10,11$ y 13 se encontraron diferencias estadísticamente significativas por sexo, mostrando que en estos ítems, los hombres tienen menores conocimientos que las mujeres o que prevalecen actitudes machistas con respecto al sexo opuesto.

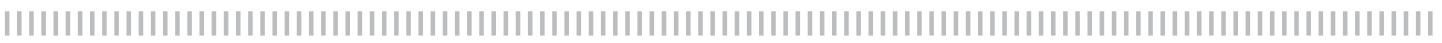

\section{Discusión}

El análisis de los datos muestra que aproximadamente la mitad de los estudiantes de educación superior en el estado de Aguascalientes han tenido relaciones sexuales, que comienzan cerca del final de su etapa adolescente; este hecho en sí mismo implica considerar a este grupo de población como de alto riesgo, sobre todo si se considera que una proporción importante de jóvenes no cuentan con los elementos formativos en sexualidad necesarios para generar procesos responsables de toma de decisiones que les permita ejercer su sexualidad adecuadamente. Es importante resaltar que ya no sólo se inician relaciones sexuales con su novio(a), sino que los y las amigos(as), así como el número de parejas sexuales, sobre todo en el sexo masculino, entran a jugar un rol importante, lo que puede marcar una diferencia sustancial, en la cual el "amor" ya no es la razón exclusiva que justificaba las relaciones sexuales.

El status laboral aunado a la carencia de información adecuada y oportuna, así como no utilizar o usar inadecuadamente los métodos anticonceptivos se refleja en el número de estudiantes que tienen hijos apenas terminada su etapa adolescente, de los cuales más de la mitad fueron no planeados; ello implica una responsabilidad mayor para estas personas, quienes además de estudiar tienen que trabajar para sostener a su nueva familia, sin contar quienes forzadamente deben dejar los estudios para dedicarse exclusivamente al trabajo, o aquellos que "obligadamente" tienen que casarse para no ser presas del estigma social, todo ello en detrimento de su formación académica e incluso truncando su proyecto de vida.

Los resultados confirman que los estudiantes hubieran preferido recibir la información sobre sexualidad de otras fuentes, principalmente de sus familiares, de médicos y maestros.

Es importante recalcar el uso del condón en una buena proporción de jóvenes, esto habla de que finalmente todas las campañas que han realizado instituciones públicas y privadas, estatales, nacionales e internacionales, han tenido eco y han contribuido a desarrollar la cultura del condón, aunque todavía les falta información sobre cómo utilizarlo adecuadamente.

En relación al uso regular de métodos anticonceptivos nos encontramos con un buen porcentaje que los usa, pero al indagar sobre su conocimiento y utilización adecuada, encontramos que les falta información co- 
rrecta que les ayude a minimizar los riesgos a los que se exponen, como en el caso de las estudiantes que se embarazaron sin desearlo y que optaron por el aborto.

Los resultados del presente estudio son similares a los encontrados en investigaciones hechas en nuestro país ${ }^{1,9-12}$, con ex- cepción de la edad de inicio de vida sexual activa, que resultó más elevada; esto se debe quizá a que los jóvenes aguascalentenses son más conservadores en el ejercicio de su sexualidad que otros de sociedades más abiertas al tema como los del Distrito Federal.

\section{Conclusiones}

a. Los estudiantes de educación superior inician relaciones sexuales entre los 17 y 18 años, en su mayoría utilizando el condón, pero desconociendo y manejando prejuicios y mitos acerca de los diferentes métodos anticonceptivos.

b. Los hombres están expuestos a mayores riesgos en el ejercicio de su sexualidad que las mujeres.

c. Un porcentaje muy importante de ellos están en situación de riesgo ante embarazos no deseados, aborto inducido, infecciones de transmisión sexual como el VIH/SIDA, un matrimonio precoz, ó la maternidad en soltería.

d. La necesidad de otorgar educación sexual está como una prioridad, antes de que los jóvenes lleguen a la Universidad.

e. La necesidad de proporcionar información a los padres de familia a través de programas educativos como el de "Escuela para Padres" sobre temas relacionados con el ejercicio de la sexualidad en los jóvenes, para que sean ellos, quienes en el seno de la familia, en un ambiente de respeto y cariño informen suficientemente a sus hijos sobre el ejercicio adecuado de su sexualidad.

\section{Bibliografía}

1. Fleiz-Bautista C., et al. Conducta sexual en estudiantes de la ciudad de México. Salud Mental. 1999 Jul; 22(4): 14 - 20.

2. Moreno S, León-Canelón M, Becerra L. Conducta sexual, conocimiento sobre embarazo y necesidades percibidas con relación a educación sexual, en adolescentes escolarizados. Cuaderno Venezolano de Sociología. 2006 octubre-diciembre; 15(4): 787 - 803.

3. Greydanus RE, Rimza ME, Matytsina L. Contraception for college students. Pediatr North Am. 2005 Feb; 52(1): 135 - 61.

4. Desjardins MF, Langlois S, Lemoyne Y. Epidemiological study on the sexuality of adolescent college students. Union Med Can. 1986 Sep; 115(9): 668 - 71.

5. Hale RW., et al. Seventeen year review of sexual and contraceptive behavior on a college campus. Am J Obstet Gynecol. 1993 Jun; 168(6): 1837 - 8.

6. Moore-Nelwin B. Parents as first sexuality information sources: Do they make a difference in daughters sexual attitudes and behavior?. Journal of Sex Education \& Therapy. 1999; 24(3): 155 - 63.
7. Millán $T$, Valenzuela $S$, Vargas NA. Reproductive health in adolescent students: knowledge, attitudes, and behavior in both sexes, in a community of Santiago. Rev Med Chil. 1995 Mar; 123(3): 368 - 75.

8. Feigenbaum $R$, Weinstein $E$, Rosen $E$. College students sexual attitudes and behaviors: implications for sexuality education. J Am Coll Health. 1995 Nov; 44(3): $112-118$.

9. Secretaría de Educación Pública. Instituto Mexicano de la Juventud. Perspectiva de la juventud en México. De: http://cendoc.imjuventud.gob.mx/investigacion/docs/Perspectiva\% 20de\% 20la\% 20juventud \% 20en\% 20M\% C3\% A9xico.pdf, enero 2008.

10. Instituto de la Juventud del Distrito Federal. Encuesta de salud sexual y reproductiva. De: http://www. jovenes.df.gob.mx/biblioDocs/02informacion/encuestaSaludSexualmayo.pdf, octubre 2007.

11. Olaíz-Fernández G., et al. Encuesta Nacional de Salud y Nutrición 2006. Resultados por entidad federativa: Aguascalientes. Cuernavaca, México: Instituto Nacional de Salud Pública, 2006.

12. Juárez $F$, Gayet $C$. Salud sexual y reproductiva de los adolescentes en México: Un nuevo marco de análisis para la evaluación y diseño de políticas. Papeles de Población. Universidad Autónoma del Estado de México, julio - septiembre 2005. 